CERN-TH/2001-078

\title{
Randall-Sundrum II Cosmology, AdS/CFT, and the Bulk Black Hole
}

\author{
Arthur Hebecker and John March-Russell \\ Theory Division, CERN, CH-1211 Geneva 23, Switzerland
}

(March 20, 2001)

\begin{abstract}
We analyse the cosmology of a brane world model where a single brane carrying the standard model fields forms the boundary of a 5-dimensional AdS bulk (the Randall-Sundrum II scenario). We focus on the thermal radiation of bulk gravitons, the formation of the bulk black hole, and the holographic AdS/CFT definition of the RSII theory. Our detailed calculation of bulk radiation reduces previous estimates to a phenomenologically acceptable, although potentially visible level. In late cosmology, in which the Friedmann equation depends linearly on the energy density $\rho$, only about $0.5 \%$ of energy density is lost to the black hole or, equivalently, to the 'dark radiation' ( $\Omega_{d, N} \simeq 0.005$ at nucleosynthesis). The preceding, unconventional $\rho^{2}$ period can produce up to $5 \%$ dark radiation $\left(\Omega_{d, N} \lesssim 0.05\right)$. The AdS/CFT correspondence provides an equivalent description of late RSII cosmology. We show how the AdS/CFT formulation can reproduce the $\rho^{2}$ correction to the standard treatment at low matter density. However, the 4-dimensional effective theory of CFT + gravity breaks down due to higher curvature terms for energy densities where $\rho^{2}$ behaviour in the Friedmann equation is usually predicted. We emphasize that, in going beyond this energy density, the microscopic formulation of the theory becomes essential. For example, the pure $\mathrm{AdS}_{5}$ and string-motivated $\mathrm{AdS}_{5} \times \mathrm{S}^{5}$ definitions differ in their cosmological implications.
\end{abstract}




\section{Introduction}

The brane world scenarios of Randall and Sundrum [1,2] have opened new perspectives in physics beyond the standard model. The one-brane scenario of [2] (RSII) appears to be particularly interesting from the point of view of gravitation and cosmology.

In RSII, the standard model fields are confined to the boundary of an infinitely extended $\mathrm{AdS}_{5}$ bulk. Four-dimensional gravity is recovered at length scales above the AdS length $\ell$. While related models with warped and non-compact extra dimensions have been considered before [3], an extensive discussion has been triggered by the specific model of [2]. An important reason for this is the proximity of this construction to recent more formal developments, such as D-branes and the AdS/CFT correspondence. On the phenomenological side, the RSII model demonstrates the extent to which gravity can be changed without violating experimental constraints. This is manifest in the existence of infinitely many, weakly coupled light modes and in the possibility of an unconventional cosmological evolution.

Although there have been many previous treatments of 'brane cosmology', the majority of these focus on either the situation for flat extra dimensions [4, which is highly constrained because of either the problem of bulk gravitons or the radion problem [5], or on the situation in RSI with the additional 'TeV-brane' and radion problems [6].

In the present paper, we develop the physical picture of the early universe in RSII, including in particular the phenomenologically relevant dynamics of bulk gravitons, the physics of the bulk black hole, the use of the AdS/CFT correspondence to make manifest some properties of the theory in the IR, and the varying early cosmology resulting from the possibility of quite different definitions of the theory in the UV.

The investigation of RSII cosmology and phenomenology was pioneered in [7,8, , , 10, and [11,12,14,13, respectively. The cosmological evolution is most conveniently discussed in terms of a moving brane with static AdS-Schwarzschild metric in the bulk. This is equivalent to the description with an expanding bulk [15. The two main unconventional cosmological features are the dark radiation (characterized by the size of the black hole in the bulk) and the $\rho^{2}$ term in the Friedmann equation. Both effects are governed by the 'AdS-cosmology scale' $M_{c}=\sqrt{M_{4} / \ell}$, where $M_{4}$ is the 4-dimensional reduced Planck mass. In the most radical scenario with $\ell \sim 1 \mathrm{~mm}$, one has a 5 -dimensional reduced Planck mass $M_{5}=\sqrt[3]{M_{4}^{2} / \ell} \simeq 1.1 \times 10^{5} \mathrm{TeV}$ and $M_{c} \simeq 0.7 \mathrm{TeV}$.

So far, much attention has been devoted to the geometrical brane dynamics and its possible extensions [16] and to the evolution of inhomogeneities in the early universe [17] (see [18 for recent reviews). However, the discussion of radiation of gravitons by the brane has been less prominent. An estimate of this effect has been given in [10]. Other, model dependent, sources for dark radiation have been discussed in [19].

We argue that graviton radiation by the hot brane will unavoidably produce a black hole in the bulk. If one assumes that the black hole was initially very small (e.g., because of inflation on the brane), then the size of the black hole is a calculable function of the reheating temperature. We present a detailed calculation of the rate at which energy is lost 
to the bulk due to graviton radiation. Our result allows for an extended $\rho^{2}$ period in the early universe. More specifically, we find that the $\rho$ period produces dark radiation corresponding to $\Omega_{d, N} \simeq 0.005$ at nucleosynthesis, while a maximally extended $\rho^{2}$ period can result in $\Omega_{d, N} \sim 0.05$. These numbers are small since, parametrically, they are inversely proportional to the number of brane degrees of freedom (this number, which is $\mathcal{O}(100)$ in the standard model, enhances the expansion rate, thus leaving less time for graviton production). The contribution of the $\rho^{2}$ period is enhanced by a factor $\sim \ln \left(T_{\max } / M_{c}\right)$ with the respect to the $\rho$ period, where $T_{\max }$ is the maximal brane temperature. It is interesting to note that gravitons emitted sufficiently early remain bound to the brane and fall into the horizon only at the end of the $\rho^{2}$ period. Our numerical results for $\Omega_{d, N}$ can be lowered or enhanced by having additional degrees of freedom on the brane or in the bulk respectively. From a phenomenological point of view, it is amusing to note that the size of $\Omega_{d}$ is potentially observable.

Gubser [10] has initiated an AdS/CFT description of RSII cosmology (see also [20]) in which the CFT corresponds to the bulk degrees of freedom. The CFT temperature, which is the bulk temperature near the brane, is responsible for the dark radiation. It is known that the leading corrections to $4 \mathrm{~d}$ gravity arising from the bulk KaluzaKlein modes are reproduced by the CFT-gravity coupling. We point out that the direct coupling of brane fields to the CFT can be responsible for the known $\rho^{2}$ corrections in the Friedmann equation. In addition, at the same densities when these corrections become relevant, higher curvature terms induced by the CFT start to affect the $4 \mathrm{~d}$ gravity theory. These effects, naively suppressed by $M_{4}$, come in so early because of the large number of degrees of freedom of the CFT. Furthermore, we emphasize that the 'UV region' $r<\ell$ of RSII is very different in the string motivated case of $\mathrm{AdS}_{5} \times \mathrm{S}^{5}$.

Our paper is organized as follows. After discussing the basic cosmological setting and the origin of the bulk black hole in Sect. 2, we derive the energy loss through graviton radiation in Sect. 3. Section 4 deals with the AdS/CFT perspective and, in particular, with the UV definition of RSII. We summarize and outline interesting open questions in Sect. 5 and present some formulae related to the graviton production rate in the Appendix.

\section{Basics of late cosmological evolution}

We start from the 5-dimensional action [2]

$$
S=\int d^{5} x \sqrt{-g_{5}}\left(\frac{1}{2} M_{5}^{3} \mathcal{R}-\Lambda_{5}\right)+\int_{\text {brane }} d^{4} x \sqrt{-g_{4}}\left(\mathcal{L}_{S M}-\Lambda_{4}\right)
$$

which includes the contribution from a 4-dimensional brane with induced metric $g_{4}$. We suppose that the standard model fields characterized by the Lagrangian $\mathcal{L}_{S M}$ are localized on this brane. The situation is further simplified by identifying the regions on both sides of the brane, i.e., imposing $Z_{2}$ orbifold boundary conditions on the 5 -dimensional spacetime. Our treatment of the dynamics of this system will follow closely the particularly clear and compact discussion of Kraus [8]. 
The motion of the brane follows from the 5-dimensional Einstein equations. It can be characterized most conveniently in terms of the extrinsic curvature $K_{\mu \nu}$ of the brane (with brane indices $\mu, \nu=0 \ldots 3$ ). $K_{\mu \nu}$, also known as the second fundamental form, can be defined as the projection of

$$
K_{M N}=\nabla_{M} n_{N},
$$

(with bulk indices $M, N=0 \ldots 4$ ) onto the brane, where $n_{N}$ is the unit normal. Then the brane motion is determined by the Israel junction condition [21] (see also [22])

$$
K_{\mu \nu}^{+}-K_{\mu \nu}^{-}=2 K_{\mu \nu}=-\frac{1}{M_{5}^{3}}\left(T_{\mu \nu}-\frac{1}{3} T_{\rho}^{\rho} g_{4, \mu \nu}\right)
$$

where the index ' + ' refers to the side of the brane to which $n^{N}$ points and the index ' - ' refers to the opposite side of the brane. The energy-momentum tensor $T_{\mu \nu}$ follows from the brane action in Eq. (11).

For negative $\Lambda_{5}$, the AdS metric

$$
d s^{2}=-\frac{r^{2}}{\ell^{2}} d t^{2}+r^{2}(d \vec{x})^{2}+\frac{\ell^{2}}{r^{2}} d r^{2},
$$

with $\ell^{2}=6 M_{5}^{3} /\left|\Lambda_{5}\right|$, represents a vacuum solution of the 5-dimensional Einstein equations. If, in addition, the 4-dimensional cosmological constant, i.e., the brane tension, satisfies the relation $\Lambda_{4}=6 M_{5}^{3} / \ell$, then the Israel junction conditions allow for an empty brane to be at rest in the above coordinates, e.g., at the position $r=R$.

In this situation, the brane observer sees 4-dimensional gravity at distance scales greater than $\ell$. The corresponding reduced Planck mass, $M_{4}=1 / \sqrt{8 \pi G_{4}}$, is fixed by the relation

$$
M_{4}^{2}=M_{5}^{3} \ell,
$$

where $M_{5}$ is the analogously defined 5-dimensional reduced Planck mass. Thus, in our following analysis, it will be convenient to consider $M_{4}$ and $\ell$ as our basic physical parameters and $M_{5}, \Lambda_{4}$ and $\Lambda_{5}$ as derived quantities.

Starting from Gauss's Theorema Egregium (see, e.g., [23]), which expresses the bulk curvature near the brane in terms of the intrinsic and extrinsic brane curvatures, and replacing the extrinsic brane curvature with the brane energy-momentum tensor according to Eq. (3), one arrives at 9] (see also [24])

$$
M_{4}^{2} \mathcal{R}_{\mu \nu}^{4}=\tau_{\mu \nu}-\frac{1}{2} \tau g_{4, \mu \nu}-\frac{1}{4 M_{c}^{4}} \tau_{\mu}^{\rho}\left(\tau_{\rho \nu}-\frac{1}{3} \tau g_{4, \rho \nu}\right)+M_{4}^{2} \delta \mathcal{R}_{\mu \rho \nu \sigma}^{5} g_{4}^{\rho \sigma} .
$$

Here $\tau_{\mu \nu}=T_{\mu \nu}+\Lambda_{4} g_{4, \mu \nu}$ is the matter contribution to the brane energy-momentum tensor, $\mathcal{R}_{\mu \nu}^{4}$ is the brane Ricci tensor and $\delta \mathcal{R}_{\mu \rho \nu \sigma}^{5}$ is the brane projection of the deviation of the bulk curvature tensor from its pure-AdS value. As will be discussed in detail below, the 'AdS-cosmology scale'

$$
M_{c}=\sqrt{M_{4} / \ell}
$$

plays a prominent role in the cosmology of the Randall-Sundrum model. It is intermediate with respect to the AdS-scale $1 / \ell$ and the 'strong gravity scale' $M_{5}=\sqrt[3]{M_{4}^{2} / \ell}$ since, in 
the physically interesting limit $1 / \ell \ll M_{4}$, one has $1 / \ell \ll M_{c} \ll M_{5}$. Equation (6) reduces to the Einstein equation for the induced brane metric in the limit where the bulk is pure AdS and energy densities on the brane are much smaller than $M_{c}^{4}$.

To discuss cosmology, we have to introduce hot matter on the brane. In this situation, the bulk solution, Eq. (4), is not general enough. The reason for this is the unavoidable graviton radiation off the brane. No matter how small the amount of energy lost to the bulk might be, it will fall deep into the AdS bulk and produce, at least if back-reaction on the metric is neglected, an arbitrarily high local energy density. The only plausible physical resolution of this problem that we are aware of is offered by the creation of a black hole in the bulk.

To see this more explicitly, consider the trajectory of a graviton radiated at time $t=0$ by a static brane at position $R$. At large $t$, this trajectory is given by

$$
r=\frac{R}{1+\left(R t / \ell^{2}\right) \sin \varphi} \simeq \frac{\ell^{2}}{t \sin \varphi},
$$

where $\varphi$ is the angle between the initial graviton momentum and the brane. (The dependence on this angle is obtained most easily by considering brane-perpendicular radiation first and then boosting the configuration along the brane.) Assume next that a 3-dimensional energy density $\Delta \rho$ is lost to gravitons with angles between $\varphi$ and $\varphi+\Delta \varphi$. At large $t$, these gravitons will be found in a slice of the AdS bulk of invariant thickness

$$
\frac{\ell}{r} \Delta r \simeq \frac{\ell}{r} \cdot \frac{\ell^{2} \cos \varphi}{t \sin ^{2} \varphi} \cdot \Delta \varphi \simeq \frac{\cos \varphi}{\sin \varphi} \cdot \ell \Delta \varphi .
$$

Note that the separation of gravitons in $r$ direction due to different times of their emission from the brane is small compared to the separation due to different angles of emission. In the rest frame of the matter on the brane, the brane-parallel components of the graviton momenta are isotropically distributed. Therefore the radiation in the above slice $\Delta r$ has its own 'rest frame'. In this frame, the momenta in $r$ direction vanish and the momenta in brane direction are blue-shifted by a factor $R / r$ with respect to their original values. Furthermore, the density in brane direction is increased by a factor $(R / r)^{3}$ due to AdS geometry. Thus, the 4-dimensional energy density of the radiation at position $r$, measured in its own rest frame, is given by

$$
\rho_{r} \simeq \frac{\Delta \rho}{(\ell / r) \Delta r}\left(\frac{R}{r}\right)^{4} \cos \varphi \simeq \frac{\Delta \rho}{\ell \Delta \varphi}\left(\frac{R}{r}\right)^{4} \sin \varphi .
$$

This local energy density diverges as $r \rightarrow 0$ and we are forced to conclude that eventually a horizon will form, hiding any possible super-Planck-scale effects from our view. Thus, the relevant bulk metric is given by the AdS-Schwarzschild solution

$$
d s^{2}=-f(r) d t^{2}+r^{2}(d \vec{x})^{2}+f(r)^{-1} d r^{2}
$$

with

$$
f(r)=\frac{r^{2}}{\ell^{2}}\left(1-\frac{r_{h}^{4}}{r^{4}}\right)
$$


where $r_{h}$ is the position of the black hole horizon.

Now the Israel junction conditions or, equivalently, Eq. (6) lead to the following equation of motion for the brane:

$$
3 M_{4}^{2}\left(\frac{\dot{R}}{R}\right)^{2}=\rho\left(1+\frac{\rho}{12 M_{c}^{4}}\right)+3 M_{c}^{4}\left(\frac{r_{h}}{R}\right)^{4},
$$

where $\dot{R}=d R / d \tau$ and $\tau$ is the proper time of the brane observer. Since the brane metric is given by

$$
d s_{b}^{2}=-d \tau^{2}+R^{2}(d \vec{x})^{2},
$$

the small- $\rho$ and small- $r_{h}$ limit of Eq. (13) reproduces the familiar 4-dimensional Friedmann equation. Deviations are characterized by $M_{c}$. On the one hand, $M_{c}$ determines the scale at which the $\rho^{2}$ term in Eq. (13) becomes important. On the other hand, it sets the scale of the last term on the rhs of Eq. (13). This term, which contributes to the expansion rate like an energy density $\rho_{d}$ of 'dark radiation', is $\sim M_{c}^{4}$ if the brane is near the black hole horizon, $R \sim r_{h}$. Furthermore, as will be discussed in detail in the next section, the radiation of bulk gravitons competes with the Hubble expansion rate $H=\dot{R} / R$ at brane temperatures $T \gtrsim M_{c}$.

Let us add a comment concerning the position of the horizon (or, equivalently, the size of the black hole) characterized by $r_{h}$. As can be seen from Eqs. (11)-(14), the absolute value of this quantity is not physical. In fact, nothing changes if one rescales the bulk coordinates and the positions of brane and horizon according to

$$
x^{\mu} \rightarrow \alpha x^{\mu} \quad, \quad r \rightarrow r / \alpha \quad \text { and } \quad R \rightarrow R / \alpha \quad, \quad r_{h} \rightarrow r_{h} / \alpha
$$

with some real number $\alpha>0$. The important physical parameter is the ratio $r_{h} / R$ (see Eq. (13) ). Note furthermore that this degeneracy is lifted if one considers closed or open geometries, where $f(r)=k+r^{2} / \ell^{2}\left(1-r_{h}^{2} / r^{2}\right)$ with $k= \pm 1$. However, since we are only interested in the early universe, we will primarily consider the flat case with $k=0$.

\section{Dark radiation and graviton emission into the bulk}

Let us start with the cross section for the production of bulk gravitons by matter on the brane. If the cms-energy of the collision is large compared to particle masses, $\sqrt{s} \gg m$, and to the typical scale of the AdS-space curvature, $\sqrt{s} \gg 1 / \ell$, the only relevant scales in the process are $\sqrt{s}$ and the 5 -dimensional Planck mass. Since the cross section has to be proportional to the 5 -dimensional gravitational coupling, we expect $\sigma(s) \sim \sqrt{s} / M_{5}^{3}$ on dimensional grounds. ${ }^{1}$

\footnotetext{
${ }^{1}$ This result differs from what is implied by Eq. (33) of [10] (see also our Eq. (19)). The discrepancy arises because the estimate of Ref. [10] is based on the leading corrections to 4-dimensional gravity. By contrast, we find that the relevant regime is $\sqrt{s} \gg 1 / \ell$, i.e., truly 5-dimensional gravity. As a result, we get weaker bounds and high allowed brane temperatures.
} 
The exact results, which are derived in the Appendix utilizing previous work of [14, 25. can be summarized by writing

$$
\sigma_{i}(s)=c_{i} \frac{\sqrt{s}}{M_{5}^{3}} .
$$

The appropriate numerical constants for the cases of scalars, vector particles and fermions (with initial-state spin averaging included) are given by

$$
c_{s}=\frac{1}{12} \quad, \quad c_{v}=\frac{1}{4} \quad, \quad c_{f}=\frac{1}{16}
$$

In a hot plasma, the reaction rate per 3 -volume is obtained by thermally averaging the cross section $\sigma$ for the process under consideration and multiplying it with the squared number density $n$ of the initial state particles (see, e.g., Ref. [26]). Analogously, the total rate of energy loss due to bulk graviton radiation is obtained by thermally averaging the product of cross section and lost energy (to avoid double counting the number of interactions between identical particles, a factor $1 / 2$ has to be included):

$$
\Delta \dot{\rho}=-\frac{1}{2}\left\langle\sigma v_{\mathrm{rel}} \cdot E\right\rangle n^{2}=-\frac{1}{2} \int d^{3} p_{1} d^{3} p_{2} f\left(E_{1}\right) f\left(E_{2}\right) \sigma v_{\mathrm{rel}} \cdot\left(E_{1}+E_{2}\right) .
$$

Here $f(E)=\kappa(2 \pi)^{-3}(\exp (E / T) \pm 1)^{-1}$ is the distribution function for bosons (minus sign) or fermions (plus sign) with $\kappa$ spin degrees of freedom and $v_{\text {rel }}$ is the relative velocity of the colliding particles. Using the cross section of Eq. (16) and setting particle masses to zero, one finds

$$
\Delta \dot{\rho}=-\frac{2 T^{8} c_{i}}{5 \pi^{4} M_{5}^{3}} \kappa_{i}^{2} \Gamma\left(\frac{7}{2}\right) \zeta\left(\frac{7}{2}\right) \Gamma\left(\frac{9}{2}\right) \zeta\left(\frac{9}{2}\right),
$$

in the bosonic case. An additional factor $a_{f}=\left(1-2^{-5 / 2}\right)\left(1-2^{-7 / 2}\right) \simeq 0.750$ arises in the fermionic case.

Summing the different particle species and normalizing to the total energy density of a relativistic gas one obtains

$$
\frac{\Delta \dot{\rho}}{\rho}=-C \frac{T^{4}}{M_{5}^{3}},
$$

where

$$
C=0.574 \cdot \frac{g_{s} c_{s}+2 g_{v} c_{v}+2 g_{f} a_{f} c_{f}}{g_{s}+g_{v}+(7 / 8) g_{f}}
$$

for $g_{s}$ scalar, $g_{v}$ vector, and $g_{f}$ fermionic degrees of freedom. The factors 2 in front of $g_{v}$ and $g_{f}$ are due to the 2 spin degrees of freedom of massless vector particles and fermions. In the standard model, $g_{s}=4, g_{v}=24$ and $g_{f}=90$, leading to $C=0.112$.

At sufficiently late times, the expansion of the universe is governed by 4-dimensional gravity. To be more specific, this period is characterized by $1 / H \gg \ell$ or $\rho \ll M_{c}$, which means that the term linear in $\rho$ dominates the rhs of Eq. (13) (' $\rho$ period'). In this situation, effective 4-dimensional energy-momentum conservation ensures that the loss of energy-density on the brane is equal to the gain of dark energy-density $\rho_{d}$,

$$
\Delta \dot{\rho}+\Delta \dot{\rho}_{d}=0
$$


where both $\rho$ and $\rho_{d}$ scale like radiation: $\dot{\rho}=-4 H \rho+\Delta \dot{\rho}$ and $\dot{\rho}_{d}=-4 H \rho_{d}+\Delta \dot{\rho}_{d}$. Thus, even if $\rho_{d}=0$ at some initial time $\tau_{1}$, it will develop a non-zero late-time value characterized by $\Omega_{d}=\rho_{d} /\left(\rho+\rho_{d}\right)$. If $\rho_{d} \ll \rho$, we have

$$
\Omega_{d}=\int_{\tau_{1}}^{\infty} d \tau\left(-\frac{\Delta \dot{\rho}}{\rho}\right),
$$

where the integrand is given by Eq. (20). Employing the relation $\rho=g_{*}\left(\pi^{2} / 30\right) T^{4}$ (where $g_{*}$ is the effective number of degrees of freedom, $\left.g_{*}=g_{s}+g_{v}+(7 / 8) g_{f}\right)$, the temperature factor $T^{4}$ can be expressed through $\rho$. Now the integral in Eq. (23) is easily performed, giving the result

$$
\Omega_{d}=\frac{15 C}{g_{*} \pi^{2}} \sqrt{\frac{3 \rho_{1}}{M_{c}^{4}}},
$$

where $\rho_{1}$ is the radiation density at $\tau=\tau_{1}$. The largest value of $\rho_{1}$ compatible with linear $\rho$ behaviour is $\rho_{1} \simeq 12 M_{c}^{4}$ (cf. Eq. (13) $)$, leading to

$$
\Omega_{d}=\frac{90 C}{g_{*} \pi^{2}} .
$$

Assuming that the evolution of the universe after the decoupling of bulk gravitons (which is complete soon after the beginning of the $\rho$ period) respects entropy conservation, one finds that at the time of nucleosynthesis

$$
\Omega_{d, N}=\left(\frac{g_{*, N}}{g_{*}}\right)^{1 / 3} \Omega_{d} .
$$

Here $g_{*, N} \simeq 10.75$ is the number of light degrees of freedom at nucleosynthesis. Thus, one arrives at the result that bulk graviton production during the complete $\rho$ period only gives rise to $\Omega_{d}=0.0044$, i.e., somewhat less than $0.5 \%$ of energy density in dark radiation. The smallness of this contribution is a direct result of the large number of light fields on the brane, which leads to a fast expansion and a correspondingly fast cooling of the universe.

The above result is encouraging for two reasons. First it implies that late cosmological evolution in the RSII model is quite safe (although not without a potential signature in future accurate CMBR measurements) even assuming the most extreme values for $\ell$ and $M_{5}$. Second it allows for a cosmological $\rho^{2}$ period with hot matter on the brane. However, also during the $\rho^{2}$ period matter is lost to dark radiation and we have to calculate the resulting contribution to $\Omega_{d}$. Since we do not have effective 4-dimensional gravity during the $\rho^{2}$ period, the energy lost by the brane is not necessarily the same as the energy found at late times in dark radiation, $\Omega_{\text {lost }} \neq \Omega_{d}$. Equation (23) is still valid, but with the lhs replaced by $\Omega_{\text {lost }}$ and with $\tau_{1}$ as the upper limit of integration,

$$
\Omega_{\text {lost }}=\int_{\tau_{0}}^{\tau_{1}} d \tau\left(-\frac{\Delta \dot{\rho}}{\rho}\right) .
$$

Here $\tau_{0}$ is the earliest time at which our analysis is valid, e.g., the time of reheating. 
The Friedmann equation in the $\rho^{2}$ period,

$$
6 M_{5}^{3} H=\rho,
$$

which follows from Eq. (13) in the limit $\rho \gg M_{c}^{4}$, implies that $\rho \sim 1 / \tau$ and $H=(1 / 4 \tau)$. In this situation, Eqs. (20) and (27) give

$$
\Omega_{\text {lost }}=\frac{45 C}{g_{*} \pi^{2}} \ln \left(\rho_{0} / \rho_{1}\right) \simeq \frac{90 C}{g_{*} \pi^{2}} \cdot \frac{1}{3} \ln \left(M_{4} \ell / 12^{3 / 2}\right) .
$$

The last approximate equality follows by assuming $\rho_{0} \simeq M_{5}^{4}$, the highest density compatible with weakly interacting gravity. This result shows an enhancement by a potentially large factor $(1 / 3) \ln \left(M_{4} \ell\right)$ compared to Eq. (25).

We still have to adress the question of how $\Omega_{\text {lost }}$ at the $\rho^{2}$ period translates into $\Omega_{d}$ at late times. This is most easily done from the brane perspective. During the $\rho^{2}$ period, the brane moves with almost speed-of-light through the AdS bulk (a natural bulk rest frame can be defined by the black-hole horizon or, equivalently, by the late-time limit, where the brane is almost at rest). The (absolute) acceleration of the brane can be calculated using the standard formula

$$
a^{M}=u^{N} \nabla_{N} u^{M},
$$

where $u^{M}=d x^{M} / d \tau$ is the velocity of a particle at rest on the brane. Multiplying this acceleration with the brane normal vector $n^{M}$ (which is, in fact, the direction of the acceleration) and making use of the orthogonality relation $n \cdot u=n_{M} u^{M}=0$, one derives

$$
a=n \cdot a=-u^{M} u^{N} \nabla_{M} n_{N} .
$$

This is precisely the $\tau \tau$ component of the extrinsic curvature $K_{\mu \nu}$. Using the Israel junction condition, Eq. (3), and defining $n^{M}$ to point into the direction of increasing $r$, the acceleration is found to be

$$
a=-\frac{1}{2 M_{5}^{3}}\left(\Lambda_{4}+\rho\right) .
$$

Notice first that this provides a nice physical interpretation of the Israel junction condition: the brane motion is determined by the brane acceleration, which, in turn, is determined by the energy density on the brane. Furthermore, deep in the $\rho^{2}$ period, the brane can be thought of as accelerating into the direction of decreasing $r$ with $a \sim \rho / M_{5}^{3}$. This implies that gravitons radiated by the brane are accelerated towards the brane with the same acceleration $a$. Since, in a thermalized situation, gravitons are emitted with smooth angular distribution, most of them will fall back onto the brane after having reached a maximal distance

$$
d \sim \frac{1}{a} \sim \frac{M_{5}^{3}}{\rho} .
$$

Clearly, this discussion becomes invalid if $d \gtrsim \ell$, since then the AdS curvature takes over and determines the future path of the graviton. In this situation, the graviton will not return to the brane but fall into the black hole horizon. 
The critical value of $\rho$ is determined Eq. (33) with $a \sim 1 / \ell$,

$$
\rho \sim \frac{M_{5}^{3}}{\ell} \sim M_{c}^{4}
$$

This means that virtually no gravitons leave the vicinity of the brane during the $\rho^{2}$ period. Instead, the gravitons radiated by the brane remain gravitationally bound to the brane and fall into the black hole only at the end of the $\rho^{2}$ period, when the motion of the brane becomes non-relativistic.

Between its initial emission and its final fall into the horizon, each graviton can bounce off the brane many times. During this process, the graviton momentum parallel to the brane remains unchanged except for the trivial redshift factor associated with the AdS geometry. However, the momentum transverse to the brane decreases dramatically. This is seen most easily by observing that, in the black hole frame, the graviton is reflected many times by the retreating brane, losing part of its momentum in the $r$ direction with each reflection.

Thus, we expect that the $\rho^{2}$ period contributes an amount $\Omega_{d}=\alpha \Omega_{\text {lost }}$ (with $\alpha<1$ ) to the late-time dark energy fraction. A lower bound on the constant $\alpha$ can be derived by assuming that only the energy going into the brane-parallel motion of the radiated gravitons will survive the multiple reflection phase and make it into the black hole horizon. This corresponds to replacing the factor $\left(E_{1}+E_{2}\right)$ in Eq. (18) by $\left|\vec{p}_{1}+\vec{p}_{2}\right|$. Recalling that $\sigma \sim \sqrt{s}$ and $v_{\text {rel }}=1-\cos \theta$, one finds

$$
\alpha=\frac{\int d^{3} p_{1} d^{3} p_{2} f\left(E_{1}\right) f\left(E_{2}\right) \sqrt{s}(1-\cos \theta)\left|\vec{p}_{1}+\vec{p}_{2}\right|}{\int d^{3} p_{1} d^{3} p_{2} f\left(E_{1}\right) f\left(E_{2}\right) \sqrt{s}(1-\cos \theta)\left(E_{1}+E_{2}\right)} .
$$

To get an estimate, we use the relation

$$
\left|\vec{p}_{1}+\vec{p}_{2}\right|=\sqrt{E_{1}^{2}+E_{2}^{2}+2 E_{1} E_{2} \cos \theta} \geq\left(E_{1}+E_{2}\right) \sqrt{(1+\cos \theta) / 2},
$$

where $\theta$ is the angle between $\vec{p}_{1}$ and $\vec{p}_{2}$ and the equality is realized for $E_{1}=E_{2}$. Since $\sqrt{s} \sim \sqrt{1-\cos \theta}$ and since the angular integration is performed with the measure $d(\cos \theta)$, the following bound can be derived:

$$
\alpha>\frac{\int_{-1}^{1} d(\cos \theta) \sqrt{(1+\cos \theta) / 2}(1-\cos \theta)^{3 / 2}}{\int_{-1}^{1} d \cos \theta(1-\cos \theta)^{3 / 2}}=\frac{5 \pi}{32} .
$$

Thus, we find $0.5 \lesssim \alpha<1$, and the maximally extended $\rho^{2}$ period produces a dark energy contribution

$$
\Omega_{d}=\frac{90 C}{g_{*} \pi^{2}} \cdot \frac{\alpha}{3} \ln \left(M_{4} \ell / 12^{3 / 2}\right),
$$

where $C$ is defined in Eq. (21). Even for the conservative number $\alpha \simeq 0.5$, this clearly dominates over the contribution from the $\rho$ period and corresponds, in the most optimistic scenario with $\ell \simeq 1 \mathrm{~mm}=1 /(0.2 \mathrm{meV})$, to $\Omega_{d, N} \simeq 0.05$. Such a value is marginally consistent with present nucleosynthesis bounds and would probably be visible in future CMBR analyses. 
As we discuss in the next section, the role of the black hole in late cosmology has a nice interpretation in the AdS/CFT correspondence. However, the $\rho^{2}$-period depends crucially on which definition of RSII cosmology is chosen - that inherited from the AdS/CFT correspondence, or the above naive 'brane in $\mathrm{AdS}_{5}$ ' definition.

\section{The AdS/CFT definition}

Many of the confusions concerning the early cosmology of RSII stem from the fact that there are various definitions of the theory, which accord with each other in the IR, but which can differ in their high-energy and high-temperature behaviour. The first definition is that employed by RSII and is the one used in the previous sections. Specifically, this involves a brane whose motion continues to be well-described by five-dimensional gravity at length scales below the AdS length $\ell$, the 5 d gravity description only breaking down at the fundamental $5 \mathrm{~d}$ Planck length $1 / M_{5}$.

The second definition, which we now discuss, arises from the AdS/CFT correspondence [27,28, 29]. Although more complicated in detail, this definition has the significant advantage that it is embedded in a richer context, allowing in principle a self-consistent discussion of high-energy (and high-temperature) effects via the connection to string theory.

Let us recall some basic aspects of the AdS/CFT correspondence in the most studied case. IIB string theory on $\mathrm{AdS}_{5} \times \mathrm{S}^{5}$ is conjectured to be equivalent to an $\mathcal{N}=4$ supersymmetric Yang-Mills conformal field theory on the boundary of $\mathrm{AdS}_{5}$. Defining the 't Hooft coupling of the boundary $S U(N)$ SYM theory $\lambda=g_{Y M}^{2} N$, the relation between the parameters of the theories is

$$
g_{Y M}^{2}=4 \pi g_{s} \quad, \quad\left(\frac{\ell}{\ell_{s}}\right)^{4}=\left(\frac{R_{S^{5}}}{\ell_{s}}\right)^{4}=\lambda
$$

where $g_{s}$ and $\ell_{s}$ are the string coupling and length, and $R_{S^{5}}$ is the radius of the $S^{5}$. That the boundary of the full 10-dimensional space is just 4-dimensional is easily seen from inspecting the $\mathrm{AdS}_{5} \times \mathrm{S}^{5}$ metric

$$
d s^{2}=\frac{\ell^{2}}{z^{2}}\left\{d z^{2}+d x^{2}+z^{2} d \Omega_{5}^{2}\right\}
$$

in the limit $z \rightarrow 0$. Note that the radius of the $S^{5}$ part of the background, as measured by an observer located at $z=\ell$, is $\ell$. This feature is quite generic, applying to the broad class of backgrounds of the form $\mathrm{AdS}_{5} \times \mathrm{M}_{5}$, since it derives from the ballancing of curvature contributions from the $\mathrm{AdS}_{5}$ and $\mathrm{M}_{5}$ in the bulk equations of motion.

The precise fashion in which these two theories correspond is most easily described in terms of the generating functional of correlation functions for the boundary theory

$$
Z[\psi(x)]=\left\langle\exp \int d x \psi(x) \mathcal{O}(x)\right\rangle
$$


where $\mathcal{O}(x)$ are a complete set of operators of the boundary theory, and $\psi$ are, for the moment, just the associated sources. The proposal of Refs. [29, 28, is to identify $Z[\psi(x)]$ with the functional integral of the $\mathrm{AdS}_{5} \times \mathrm{S}^{5}$ theory, with specified boundary behaviour for the fields:

$$
Z[\psi(x)]=\left.\int[d \Psi(z, x)] \exp \left(-S_{\mathrm{IIB}}[\Psi(z, x)]\right)\right|_{\Psi(z \rightarrow 0, x) \rightarrow \psi(x)} .
$$

In the limit of large $N$ and large 't Hooft coupling $\lambda$, the bulk IIB string theory goes over to its (super)gravity limit in the tree approximation. In fact, a similar correspondence with a $4 \mathrm{~d}$ boundary CFT is believed to apply much more generally for any gravity theory formulated on $\mathrm{AdS}_{5}$. In particular we will, for simplicity, consider the nonsupersymmetric case in the following.

It is important that, because of the short-distance singularities of the correlators, the above expressions must be regulated so that they are well-defined. A natural way of doing this is to impose the boundary conditions at a finite cutoff $z=z_{c}$ rather than at $z=0$. By the Weyl rescaling symmetry of the coordinates $(z, x)$, changes in the $z$ coordinate of $\mathrm{AdS}_{5}$ can be reinterpreted as changes in the energy scale of the CFT process under consideration, with $z \rightarrow 0$ corresponding to the UV limit of the boundary conformal theory. Thus the cutoff at $z=z_{c}$ implements a UV regulation of the boundary theory. In order to get a well-defined limit as the cutoff is removed, $z_{c} \rightarrow 0$, the boundary theory must be supplemented by counterterms depending on the boundary values of the bulk fields. Moreover, the cutoff at $z=z_{c}$ also allows the normalizability of the bulk AdS graviton zero mode, the existence of such a mode implying that the regulated boundary theory now also couples to dynamical gravity. In the limit of $z_{c} \rightarrow 0$, the boundary theory becomes a pure CFT decoupled from gravity, the effective Planck mass of the boundary theory $M_{4}$ going to infinity as the graviton zero mode becomes non-normalizable.

In the specific case we are interested in, this cutoff is implemented by a physical 'Planck brane' located at $z_{c}$. This Planck brane provides a physical cutoff for the bulk theory and the theory on the Planck brane is a 4-dimensional CFT coupled to dynamical 4-dimensional gravity arising from the graviton zero mode. The CFT degrees of freedom are just the new expression of the bulk Kaluza-Klein modes of the graviton in this $4 \mathrm{~d}$ 'holographic' approach.

Following Refs. 30,10,12, it is useful to be more explicit about the result of this procedure in the simple case where the only bulk field is the 5-dimensional metric $g_{5}(x, z)$, with boundary value $g_{4}(x)$ at the Planck (regulator) brane at $z_{c}{ }^{2}$ The boundary operator for which $g_{4}$ is the source is just the energy-momentum tensor $T_{\text {CFT }}^{\mu \nu}$ of the CFT, so the integration over all bulk metrics will lead to the generating functional $Z\left[g_{4}\right]=\left\langle\exp \left(-\int g_{4, \mu \nu} T_{\mathrm{CFT}}^{\mu \nu}\right)\right\rangle_{\mathrm{CFT}}$ for correlation functions of $T_{\mathrm{CFT}}$. In addition, counterterms in the boundary metric $g_{4, \mu \nu}$ must be added. Together this leads to a boundary

\footnotetext{
${ }^{2}$ In addition, we suppose that there are fields $\varphi$ localized to the Planck brane which represent the standard model degrees of freedom. In general, string theory imposes strong consistency requirements relating the spectrum of brane-localized fields to the bulk field content, so our assumption of $g_{5}$ as the sole bulk field is not really justified. However it is sufficient to illustrate the point at hand.
} 
theory described by the effective action

$$
S_{4 \mathrm{~d}}\left[g_{4}, \varphi\right]=\int d^{4} x \sqrt{g_{4}}\left\{\mathcal{L}(\varphi)-\frac{1}{2} M_{4}^{2} \mathcal{R}-b_{4} \mathcal{R}_{2}+\ldots\right\}
$$

Here $\mathcal{R}_{2}=-\mathcal{R}^{\mu \nu} \mathcal{R}_{\mu \nu} / 8+\mathcal{R}^{2} / 24$ is the leading higher-derivative pure metric piece of the counterterm action, and $b_{4}=c f\left(z_{c}\right)$ where $f\left(z_{c}\right)$ is a function that has a $\log \left(z_{c}\right)$ singularity as $z_{c} \rightarrow 0$, and $c=2 \pi^{2}\left(M_{5} \ell\right)^{3}$ is the central charge of the boundary CFT.

At long distances this effective action correctly reproduces the gravitational potential between two masses located on the Planck brane including leading corrections:

$$
V(r)=\frac{m_{1} m_{2}}{M_{4}^{2}}\left(\frac{1}{r}+a \frac{\ell^{2}}{r^{3}}+\ldots\right),
$$

for $r \gg \ell$, and $a$ a numerical coefficient. In the original bulk $\mathrm{AdS}_{5}$ picture, the $1 / r^{3}$ correction is due to the exchange of the continuous spectrum of Kaluza-Klein modes, while in the boundary CFT + 4-dimensional gravity description, the correction is due to the two-point function of the CFT energy-momentum tensor with two external $4 \mathrm{~d}$ graviton propagators $\left(1 / p^{2}\right)\langle T(p) T(-p)\rangle\left(1 / p^{2}\right) \sim \log p^{2}$. Recall that the two-point function of $T_{\mathrm{CFT}}$ satisfies

$$
\langle T(p) T(-p)\rangle \sim c p^{4} \log p^{2}
$$

with $c$ the central charge.

Note that an inspection of the calculation of Ref. [29] shows that for fixed 4d Planck mass, $M_{4}$, the central charge $c / 2 \pi^{2}=\left(M_{5} \ell\right)^{3}=\left(M_{4} \ell\right)^{2}$ of the boundary CFT depends only on the geometry of the $\mathrm{AdS}_{5}$ (through the parameter $\ell$ ). In particular, for equal values of $\ell$, the two theories defined by taking the geometry to be respectively either $\mathrm{AdS}_{5}$ or $\mathrm{AdS}_{5} \times \mathrm{S}^{5}$ have the same $4 \mathrm{~d} \mathrm{CFT}+4 \mathrm{~d}$ gravity description in the IR. ${ }^{3}$ Since the theories most certainly differ for length scales $r<\ell$ (the static gravitational potential behaving as $V(r) \sim 1 / r^{2}$ or $\sim 1 / r^{7}$ in the two cases), the $4 \mathrm{~d}$ CFT + gravity description must break down at $r \simeq \ell$ due to the existence of new strong couplings possibly involving new degrees of freedom.

As pointed out by Gubser [10, the CFT perspective is particularly useful for a physical understanding of the dark radiation term in the Friedmann equation. The AdSSchwarzschild geometry implies, by continuation to Euclidean space, a black hole temperature $T_{\mathrm{BH}}=r_{h} / \pi \ell^{2}$. This corresponds to a local temperature $T(r)=T_{\mathrm{BH}} / \sqrt{f(r)}$ in the bulk 31]. For $R \gg r_{h}$, the temperature near the brane is $T(R) \simeq r_{h} /(\pi \ell R)$. Thus, the brane observer interprets the CFT, represented by the bulk degrees of freedom, as being heated to this temperature. At weak coupling, the energy density of the heated CFT is given by $\rho=2 \pi^{2} c T^{4}$. Taking into account the famous factor $3 / 4$ (see, e.g., 32]), which is due to strong coupling effects in the CFT, one finds

$$
\rho=\frac{3 \pi^{2}}{2} c[T(R)]^{4},
$$

\footnotetext{
${ }^{3}$ In fact, because of the isometries of $\mathrm{S}^{5}$, the IR CFT of the $\mathrm{AdS}_{5} \times \mathrm{S}^{5}$ has additional conserved currents. However, the central charge and thus the late cosmology of the two models is the same (cf. Eq. (46) ).
} 
which is precisely the dark radiation term of Eq. (13). Note that, phenomenologically, $T(R)$ has to be much smaller than the standard model temperature on the brane. Otherwise, the dark radiation completely dominates the total energy density due to the large number of degrees of freedom of the CFT.

Near the horizon, $f(r)$ goes to zero and the local bulk temperature diverges. Nevertheless, the brane observer in a cosmological setting sees an effective bulk temperature $T=r_{h} /(\pi \ell R)$, since the red-shift factor from the brane motion in the blackhole rest frame compensates the singular behaviour of $1 / \sqrt{f(r)}$. We have, at present, no deeper understanding of this intriguing fact. Note, however, that the naive formula $T(r)=T_{\mathrm{BH}} / \sqrt{f(r)}$, which underlies the above discussion, should in itself be questioned near the horizon, since it is in principle affected by the ambiguities of the definition of the black-hole vacuum state (cf. 33]).

In the cosmological context of the earlier sections, an immediate question is if the $\rho^{2}$ correction in the Friedmann equation (13) is similarly reproduced by the AdS/CFT description Eq.(43). At first glance the answer appears to be no, as the $\varphi$-matter energymomentum tensor derived from $\mathcal{L}(\varphi)$ couples linearly with $g_{4, \mu \nu}$. However, the existence of the higher-derivative terms $\mathcal{R}_{2}=-\mathcal{R}^{\mu \nu} \mathcal{R}_{\mu \nu} / 8+\mathcal{R}^{2} / 24$ in the 4 d action Eq. (43) leads to an effective $\rho^{2}$ correction. ${ }^{4}$ (Recall that these higher-derivative terms are predicted by the conformal anomaly of the boundary CFT and occur with coefficient $b_{4}$ enhanced by $c$.) To see this, consider the Friedmann equations in the limit that $\rho \ll M_{c}^{4}$; in this case the Hubble length $H^{-1} \gg \ell$. Then in the leading approximation $H^{2} \sim \rho / M_{4}^{2}$, and substituting this back in to the higher-derivative $\mathcal{R}^{2}$ terms gives

$$
b_{4}\left(-\mathcal{R}^{\mu \nu} \mathcal{R}_{\mu \nu} / 8+\mathcal{R}^{2} / 24\right) \sim c H^{4} \sim \frac{\ell^{2} \rho^{2}}{M_{4}^{2}}=\frac{\rho^{2}}{M_{c}^{4}},
$$

the required $\rho^{2}$ term. The coefficient of this effective $\rho^{2}$ correction can vary, though, depending on the definition of the UV theory. For example, the boundary action Eq. (43) can contain an additional higher-dimensional counterterm that is not forbidden by any symmetry:

$$
\frac{1}{M_{4}^{4}} T_{\mu \nu}^{\mathrm{CFT}} T_{\varphi}^{\mu \nu}
$$

Such an operator directly linking the CFT with the brane-localized matter has recently been invoked 35. to explain the $1 / r^{7}$ corrections to the gravitational potential found in the Lykken-Randall 'probe-brane' scenario [36] (see also [13]). Via the use of the $T_{\mathrm{CFT}}$ two-point function, this operator in turn implies the additional interaction

$$
\Delta S_{4 \mathrm{~d}} \sim \frac{1}{M_{4}^{8}} \int d^{4} x d^{4} y T_{\varphi}(x)\left\langle T_{\mathrm{CFT}}(x) T_{\mathrm{CFT}}(y)\right\rangle T_{\varphi}(y) \sim \frac{c}{M_{4}^{8}} \int d^{4} x d^{4} y \frac{T_{\varphi}(x) T_{\varphi}(y)}{|x-y|^{8}}
$$

which, for homogeneous and isotropic radiation $T_{\varphi}=\operatorname{diag}(\rho,-\rho / 3,-\rho / 3,-\rho / 3)$, leads to a term (taking the cutoff for the Planck brane theory to be $M_{4}$ )

$$
\frac{c}{M_{4}^{4}} \rho^{2} \sim \frac{\rho^{2}}{M_{c}^{4}}
$$

\footnotetext{
${ }^{4}$ After completion of this paper we were kindly informed of the recent work of Ref. 34, where the correct coefficient of the $\rho^{2}$ term in the pure $\mathrm{AdS}_{5}$ case has been verified.
} 
on the rhs of the Friedmann equation.

Thus it is possible to account for the $\rho^{2}$ term in the modified Friedmann equation, Eq.(13), from a CFT description, although the exact coefficient of this term depends on the UV definition of the theory. In the case that the UV $(r<\ell)$ theory is just the brane in $\mathrm{AdS}_{5}$, then, by definition, the CFT version must exactly reproduce the term in Eq.(13). Alternatively if the theory is the string-motivated $\operatorname{AdS}_{5} \times \mathrm{S}^{5}$, then the $\rho^{2}$ term can receive further corrections.

Despite the existence of the $\rho^{2}$ correction to the Friedmann equation, we wish to emphasize that the unusual cosmological ' $\rho^{2}$-behavior' where this term dominates (occurring for $\rho>M_{c}^{4}$ ) is not physically accessible in the string motivated $\mathrm{AdS}_{5} \times \mathrm{S}^{5}$ definition of the theory. The reason for this is simply that for the string coupling $g_{s}<1$ (which can always be arranged by suitable strong to weak coupling duality transforms) the string scale satisfies $L_{s}^{-1} \sim g_{s}^{1 / 4}\left(M_{4} / \ell^{3}\right)^{1 / 4} \sim g_{s}^{1 / 4}\left(M_{c}^{3} / M_{4}\right)^{1 / 2} \ll M_{c}$. Thus one encounters the full string theory well before one reaches the scale at which $\rho^{2}$ behaviour begins.

\section{$5 \quad$ Summary and Comments}

In this paper we have considered some of the cosmological issues raised by taking the Randall-Sundrum II framework to describe our world. The Randall-Sundrum II proposal assumes that the SM degrees of freedom are localized on the 'Planck brane' and are therefore described by a conventional $(3+1)$-d quantum field theory up to high scales; thus the successes of, for example, supersymmetric gauge coupling unification are not necessarily spoiled. However, RSII does not itself provide a solution to the hierarchy problem - one must still assume supersymmetry or technicolour, or some other as yet undiscovered mechanism, to solve this problem for the Planck-brane QFT; on the other hand, gravity is dramatically modified leading to new effects, especially in early universe cosmology, and associated constraints or signals. RSII provides an interesting laboratory for exploring modifications to gravity and early universe cosmology.

There are two immediate new effects in RSII cosmology. The first is that the motion of our Planck brane in the AdS bulk leads to a correction to the usual Friedmann equations for the scale factor - the well known $\rho^{2}$-term. The second is the possibility of radiation into the AdS bulk, and the formation of a black hole in the AdS spacetime. Both of these issues have been previously studied. However, we argue that the amount of 'dark radiation' that could be produced during the linear-in- $\rho$ and $\rho^{2}$ epochs in the early universe was overestimated in previous treatments. This leads to the false conclusion that the Planck brane temperature could never be high enough to reach the $\rho^{2}$ region. We give a detailed account of this radiation and further show that the formation of the bulk AdS-Schwarzschild background is a necessary consequence. The numbers that we find for the dark radiation are in a region that is accessible by future more precise cosmological measurements. In particular, we predict dark radiation at nucleosynthesis with $\Omega_{d, N} \simeq$ 0.005 from the $\rho$ regime and $\Omega_{d, N} \lesssim 0.05$ from the $\rho^{2}$ regime. The precise amount of the contribution from the $\rho^{2}$ regime depends on the maximal brane temperature in the early universe. 
Another important ingredient of our paper is the extended discussion of RSII cosmology in the framework of the AdS/CFT correspondence. We show how, for small brane energy densities, the manifestly 4-dimensional CFT description can reproduce the predictions of the standard definition in which the Planck brane moves in $\mathrm{AdS}_{5}$ according to the Israel junction conditions, including the $\rho^{2}$ corrections to the Friedmann equations. However, we emphasize that there exist different definitions of the theory in the 'UV region' $r<\ell$, and the precise size of the $\rho^{2}$ corrections depends on this UV theory. In the general case, the corrections to the cosmological evolution arise both from higher-derivative terms in the curvature and higher-dimension operators connecting the on-brane matter directly to the CFT. Because of the large central charge of the CFT, the coefficient of the higher curvature terms is greatly enhanced over its naive value, indicating a precocious breakdown of the $4 \mathrm{~d}$ effective theory. In the case of the string-motivated definition on $\operatorname{AdS}_{5} \times \mathrm{M}_{5}$, the ' $\rho^{2}$-region' where the $\rho^{2}$ term dominates the evolution is not physically accessible.

There are many additional physical questions that either deserve further attention or have not been discussed at all. These include a further analysis of the near horizon, $\left(R-r_{h}\right) / r_{h} \ll 1$, behavior of the 'Israel' brane theory, and the inclusion of the many more bulk fields (in addition to the graviton) that are likely to accompany the construction of even a semi-realistic model. At low energies, the manifestly 4-dimensional CFT+gravity description is likely to be useful to analyse such questions as the production and evolution of the cosmic microwave background fluctuations. Concerning the UV definition of the RSII theory, an important general point about the string-motivated $\mathrm{AdS}_{5} \times \mathrm{M}_{5}$ definition is that it encodes a consistent description of the theory in the high-energy regime, with different physical behaviour from the standard 'Israel' definition.

\section{Acknowledgements}

We would like to thank Tony Gherghetta, Yaron Oz and Riccardo Rattazzi for helpful discussions. We are also grateful to the authors of the later paper Ref. [38] (see also [39]) for pointing out two numerical errors in the first version of this paper. Special thanks go to Lorenzo Sorbo, who helped to compare the respective calculations.

\section{Appendix}

In this appendix, we briefly describe the calculation of the cross sections for the production of bulk gravitons by scalars, fermions and vector particles on the brane. The effective 4-dimensional Lagrangian for the graviton Kaluza-Klein modes and their coupling to matter has been derived, e.g., in Ref. 14. To properly normalize the bulk excitations, one introduces a regulator brane at $r=\ell \exp (-L / \ell)$ and takes the limit $L \rightarrow \infty$ at the end. In the transverse traceless gauge, where the graviton fields $H_{(n)}^{\mu \nu}$ satisfy $H_{(n), \mu}^{\mu \nu}=0$ 
and $g_{\mu \nu} H_{(n)}^{\mu \nu}=0$, the Lagrangian reads ${ }^{5}$

$$
\mathcal{L}_{H}=-\frac{1}{2} \sum_{n}\left(H_{(n), \rho}^{\mu \nu} H_{\mu \nu}^{(n), \rho}+m_{n}^{2} H_{(n)}^{\mu \nu} H_{\mu \nu}^{(n)}\right)+T^{\mu \nu} \sum_{n} \lambda_{n} H_{\mu \nu}^{(n)},
$$

where, in the limit $m_{n} \gg 1 / \ell$,

$$
m_{n}=\frac{1}{\ell} n \pi e^{-L / \ell} \quad \text { and } \quad \lambda_{n}=\frac{1}{M_{4}} e^{-L / 2 \ell} .
$$

For each $n$, the operator $H_{\mu \nu}^{(n)}$ represents simply a massive spin-2 field with 5 physical degrees of freedom (see, e.g., [37]). Thus, introducing the 5 polarization tensors $\varepsilon_{\mu \nu}^{(i)}(q)$ (with $\varepsilon_{\mu \nu}^{(i)}(q) \varepsilon_{(j)}^{\mu \nu}(q)=\delta_{j}^{i}$ ), the final state spin sum in the production of a graviton with momentum $q$ can be taken multiplying the squared amplitude with

$$
\sum_{i} \varepsilon_{\mu \nu}^{(i)}(q) \varepsilon_{\alpha \beta}^{(i)}(q)=g_{\mu \alpha} g_{\nu \beta}-\frac{1}{3} g_{\mu \nu} g_{\alpha \beta}+\left(\text { terms } \sim q_{\mu}, q_{\nu}, q_{\alpha} \text { or } q_{\beta}\right)
$$

and summing over $\mu \nu$ and $\alpha \beta$. Terms $\sim q$ are irrelevant since the energy-momentum tensor $T^{\mu \nu}$ in Eq. A.1. is conserved.

Since we will mainly be interested in cms-energies in the TeV region, we disregard the masses of brane degrees of freedom in the following calculation. In the case of one real scalar field, the energy-momentum tensor in Eq. A.1. is given by

$$
T_{\mu \nu}=\partial_{\mu} \varphi \partial_{\nu} \varphi-\frac{1}{2} g_{\mu \nu}(\partial \varphi)^{2}
$$

Now it is straightforward to derive the Feynman rule for the $\varphi \varphi H$ vertex and to calculate the total $\varphi \varphi$ annihilation cross section after the sum over $n$ is replaced by an integral over the final state mass $m=\sqrt{s}$ according to

$$
\sum_{n} \longrightarrow \frac{\ell}{\pi} e^{L / \ell} \int d \sqrt{s}
$$

Similarly, the annihilation cross sections for two vector particles (e.g., two photons) and for a fermion-antifermion pair can be obtained by substituting the energy-momentum tensors

$$
T_{\mu \nu}=-F_{\mu \rho} F_{\nu}^{\rho}+\frac{1}{4} g_{\mu \nu} F^{2},
$$

with $F_{\mu \nu}=\partial_{\mu} A_{\nu}-\partial_{\nu} A_{\mu}$, and

$$
T_{\mu \nu}=\frac{i}{4} \bar{\psi}\left(\gamma_{\mu} \stackrel{\leftrightarrow}{\partial}_{\nu}+\gamma_{\nu} \stackrel{\leftrightarrow}{\partial}_{\mu}\right) \psi
$$

into Eq. (A.11). The resulting cross sections are given in Eqs. (16) and (17) of the main part of the paper.

\footnotetext{
${ }^{5}$ Note that we have changed the normalization of $H_{\mu \nu}$ by a factor of $\sqrt{2}$ with respect to Ref. [14] to give the graviton fields a canonical kinetic term.
} 
Note that, since we are only interested in the limit $\sqrt{s} \gg 1 / \ell$, the AdS nature of the bulk has no physical significance. Therefore, our result can be compared with the graviton production cross section on a brane in a flat, 5-dimensional bulk (the ADD scenario with one extra dimension [4] if the 5-dimensional gravitational constants are identified. Indeed, one can check explicitly that the one-graviton exchange amplitude calculated in the framework of this appendix (where $\left.i /\left(-q^{2}-m_{n}^{2}\right) \sum_{j} \varepsilon_{\mu \nu}^{(j)}(q) \varepsilon_{\alpha \beta}^{(j)}(q)\right)$ is the graviton propagator) is identical to the result of Ref. [25] (see Eqs. (69)-(71) with $\delta=1)$.

\section{References}

[1] L. Randall and R. Sundrum, Phys. Rev. Lett. 83 (1999) 3370 hep-ph/9905221.

[2] L. Randall and R. Sundrum, Phys. Rev. Lett. 83 (1999) 4690 hep-th/9906064.

[3] V. A. Rubakov and M. E. Shaposhnikov, Phys. Lett. B 125 (1983) 139;

P. van Nieuwenhuizen and N. P. Warner, Commun. Math. Phys. 99 (1985) 141;

H. Nicolai and C. Wetterich, Phys. Lett. B 150 (1985) 347;

A. Strominger, Nucl. Phys. B 274 (1986) 253;

B. de Wit, D. J. Smit and N. D. Hari Dass, Nucl. Phys. B 283 (1987) 165.

[4] N. Arkani-Hamed, S. Dimopoulos and G. Dvali, Phys. Lett. B 429 (1998) 263 hep-ph/9803315.

[5] N. Arkani-Hamed, S. Dimopoulos and G. Dvali, Phys. Rev. D 59 (1999) 086004 hep-ph/9807344;

L. J. Hall and D. Smith, Phys. Rev. D 60 (1999) 085008 hep-ph/9904267;

N. Arkani-Hamed, S. Dimopoulos, N. Kaloper and J. March-Russell, Nucl. Phys. B 567 (2000) 189 hep-ph/9903224.

[6] P. Binetruy, C. Deffayet and D. Langlois, Nucl. Phys. B 565 (2000) 269 hep-th/9905012;

J. M. Cline, C. Grojean and G. Servant, Phys. Rev. Lett. 83 (1999) 4245 hep-ph/9906523;

C. Csaki, M. Graesser, C. Kolda and J. Terning, Phys. Lett. B 462 (1999) 34 hep-ph/9906513;

C. Csaki, M. Graesser, L. Randall and J. Terning, Phys. Rev. D 62 (2000) 045015 hep-ph/9911406;

P. Kanti, I. I. Kogan, K. A. Olive and M. Pospelov, Phys. Rev. D 61 (2000) 106004 hep-ph/9912266.

[7] N. Kaloper, Phys. Rev. D 60 (1999) 123506 hep-th/9905210;

P. Binetruy, C. Deffayet, U. Ellwanger and D. Langlois, Phys. Lett. B 477 (2000) 285 hep-th/9910219; 
A. Kehagias and E. Kiritsis, JHEP9911 (1999) 022 hep-th/9910174;

E. E. Flanagan, S. H. Tye and I. Wasserman, Phys. Rev. D 62 (2000) 044039 hep-ph/9910498;

S. Mukohyama, Phys. Lett. B 473 (2000) 241 hep-th/9911165|;

J. Garriga and M. Sasaki, Phys. Rev. D 62 (2000) 043523 hep-th/9912118.

[8] P. Kraus, JHEP9912 (1999) 011 hep-th/9910149.

[9] T. Shiromizu, K. Maeda and M. Sasaki, Phys. Rev. D 62 (2000) 024012 gr-qc/9910076.

[10] S. S. Gubser, hep-th/9912001.

[11] J. Garriga and T. Tanaka, Phys. Rev. Lett. 84 (2000) 2778 hep-th/9911055.

[12] S. Giddings, E. Katz and L. Randall, hep-th/0002091.

[13] S. B. Giddings and E. Katz, hep-th/0009176.

[14] D. J. Chung, L. Everett and H. Davoudiasl, hep-ph/0010103.

[15] S. Mukohyama, T. Shiromizu and K. Maeda, Phys. Rev. D 62 (2000) 024028 hep-th/9912287.

[16] H. Stoica, S. H. Tye and I. Wasserman, Phys. Lett. B 482 (2000) 205 hep-th/0004126;

P. Bowcock, C. Charmousis and R. Gregory, Class. Quant. Grav. 17 (2000) 4745 hep-th/0007177;

A. Davis, S. C. Davis, W. B. Perkins and I. R. Vernon, hep-ph/0008132;

T. Boehm, R. Durrer and C. van de Bruck, hep-th/0102144.

[17] R. Maartens, Phys. Rev. D 62 (2000) 084023 hep-th/0004166;

D. Langlois, Phys. Rev. D 62 (2000) 126012 hep-th/0005025;

C. van de Bruck, M. Dorca, R. H. Brandenberger and A. Lukas, Phys. Rev. D 62 (2000) 123515 hep-th/0005032;

O. Seto and H. Kodama, hep-th/0012102

A. Campos and C. F. Sopuerta, hep-th/0101060.

[18] J. March-Russell, hep-ph/0012151

R. Maartens, gr-qc/0101059

H. B. Kim, hep-ph/0102182.

[19] A. Chamblin, A. Karch and A. Nayeri, hep-th/0007060;

J. Yokoyama and Y. Himemoto, hep-ph/0103115.

[20] S. Nojiri and S. D. Odintsov, JHEP0007 (2000) 049 hep-th/0006232;

L. Anchordoqui, C. Nunez and K. Olsen, JHEP0010 (2000) 050 hep-th/0007064;

I. Savonije and E. Verlinde, hep-th/0102042.

[21] W. Israel, Nuovo Cim. B 44S10 (1966) 1. 
[22] S. K. Blau, E. I. Guendelman and A. H. Guth, Phys. Rev. D 35 (1987) 1747.

[23] L.I. Nicolaescu, "Geometry of Manifolds," Singapore: World Scientific (1996).

[24] S. de Haro, K. Skenderis and S. N. Solodukhin, hep-th/0011230.

[25] G. F. Giudice, R. Rattazzi and J. D. Wells, Nucl. Phys. B 544 (1999) 3 hep-ph/9811291.

[26] M. Srednicki, R. Watkins and K. A. Olive, Nucl. Phys. B 310 (1988) 693;

E. W. Kolb and M. S. Turner, "The Early Universe" Redwood City, USA: AddisonWesley (1990) 547 p. (Frontiers in physics, 69).

[27] J. Maldacena, Adv. Theor. Math. Phys. 2 (1998) 231 hep-th/9711200.

[28] E. Witten, Adv. Theor. Math. Phys. 2 (1998) 253 hep-th/9802150.

[29] S. Gubser, I. Klebanov and A. Polyakov, Phys. Lett. B248 (1998) 105 hep-th/9802109.

[30] M. Henningson and K. Skenderis, JHEP9807 (1998) 023 hep-th/9806087.

[31] S. W. Hawking and D. N. Page, Commun. Math. Phys. 87 (1983) 577.

[32] N. Itzhaki, hep-th/9904035.

[33] D. N. Page, Phys. Rev. D 25 (1982) 1499;

N. D. Birrell and P. C. Davies, "Quantum Fields In Curved Space," Cambridge, Uk: Univ. Pr. (1982).

[34] T. Shiromizu and D. Ida, hep-th/0102035.

[35] N. Arkani-Hamed, M. Porrati and L. Randall, hep-th/0012148.

[36] J. Lykken and L. Randall, JHEP0006 (2000) 014 hep-th/9908076.

[37] M. J. Veltman, In *Les Houches 1975, Proceedings, Methods In Field Theory*, Amsterdam 1976, 265-32\%.

[38] D. Langlois, L. Sorbo and M. Rodriguez-Martinez, Phys. Rev. Lett. 89 (2002) 171301 arXiv:hep-th/0206146.

[39] D. Langlois and L. Sorbo, arXiv:hep-th/0306281. 